\title{
Temperament and Personality Traits in Patients with Chronic Spontaneous Urticaria: A Cross-Sectional Study
}

\author{
Maryam Khoshkhui ${ }^{1}$, Maedeh Kamrani (iD ${ }^{2,3}$, Maryam Emadzadeh (iD ${ }^{4}$, Zahra Jafari ${ }^{1}$ and Farhad \\ Faridhosseini (iD ${ }^{2, *}$ \\ ${ }^{1}$ Department of Immunology, Allergy Research Center, Mashhad University of Medical Sciences, Mashhad, Iran \\ ${ }^{2}$ Department of Psychiatry, Faculty of Medicine, Mashhad University of Medical Sciences, Mashhad, Iran \\ ${ }^{3}$ Psychiatry and Behavioral Sciences Research Center, Mashhad University of Medical Sciences, Mashhad, Iran \\ ${ }^{4}$ Clinical Research Unit, Faculty of Medicine, Mashhad University of Medical Sciences, Mashhad, Iran \\ "Corresponding author: Psychiatry and Behavioral Sciences Research Center, Mashhad University of Medical Sciences, Mashhad, Iran. Email: faridhoseinif@mums.ac.ir
}

Received 2021 June 23; Revised 2021 October 08; Accepted 2021 December 10.

\begin{abstract}
Background: Considering the psychobiological aspects of various dermatological diseases and the role of personality traits in the development of chronic skin diseases such as chronic urticaria, the present study aimed to assess the personality traits of patients with chronic spontaneous urticaria (CSU) compared to healthy individuals.

Methods: In this cross-sectional study during 2016 - 2018, all patients with CSU who referred to Asthma and allergic diseases clinic of Ghaem hospital, Center of reference and excellence in urticaria (UCARE), considered as research community, 100 patients with CSU were selected using the convenience sampling method. Moreover, 100 healthy participants were selected as the control group. Research instruments were Temperament and Character Inventory (TCI) and a demographic checklist. Data were analyzed using descriptive statistics, $t$-test, and chi-square test using SPSS Software version 23.

Results: The patients had higher scores in novelty-seeking $(\mathrm{P}=0.041)$ and harm avoidance $(\mathrm{P}=0.015)$, while the healthy individuals had higher scores in self-directedness $(\mathrm{P}=0.003)$ and cooperativeness $(\mathrm{P}=0.001)$. Moreover, male patients had higher scores in novelty-seeking $(\mathrm{P}=0.006)$ and reward dependence $(\mathrm{P}=0.013)$; however, female patients had higher scores in self-transcendence $(\mathrm{P}$ $=0.001)$ and cooperativeness $(\mathrm{P}=0.019)$. Furthermore, there was a correlation between the disease duration of with reward dependence, self-directedness, and self-transcendence.

Conclusions: Personality traits seem to be associated with CSU. In this regard, patients with chronic spontaneous urticaria had higher scores in novelty-seeking and harm avoidance and lower scores in self-directedness and cooperativeness than healthy individuals.
\end{abstract}

Keywords: Chronic Spontaneous Urticaria, Personality, Temperament, Trait

\section{Background}

Chronic urticaria is a multi-etiological condition which in many cases it has not specific etiology $(1,2)$. Chronic urticaria is defined as the occurrence of wheals, angioedema, or both for more than 6 weeks. It is divided to physical inducible or spontaneous urticaria (3).

Regarding pathophysiology, histamine, inflammatory mediators, prostaglandins, and leukotrienes are involved in the clinical manifestation of chronic spontaneous urticarial (CSU), and unarguably various pathologic conditions can trigger the release of these mediators (4-7). Furthermore, studies have documented that substance P was higher among chronic urticaria patients than healthy subjects (8). The prevalence rate of chronic spontaneous urticaria is $0.5-2.3 \%$, and its lifetime prevalence is $1.8 \%$ in dif- ferent countries $(9,10)$.

Studies have revealed an unneglectable relationship between skin disorders and psychosocial variables. Skin lesions can affect the quality of life (11) and patients' psychological conditions. Moreover, the psychological stressors, in turn, can aggravate skin diseases. This issue develops a novel approach, called psychodermatology (combination of dermatology, psychiatry, and psychology) (12, 13). It is estimated that the prevalence of psychiatric problems in dermatological patients is $30-60 \%$ (14). Psychiatric comorbidities in CSU patients, including depression and anxiety, are associated with prolonged inflammatory processes (15).

Considering the existing evidence, patients with dermatological diseases have a higher level of psychological 
problems (16) such as pathological worry (17), depression, and anxiety (18). Further, some personality traits are accompanied with the development of skin diseases such as atopic dermatitis (19), psoriasis (20), essential hyperhidrosis (21), and CSU (22).

\section{Objectives}

Regarding the biological models of human behaviors, Cloninger developed a questionnaire to measure temperament and character (23). Some studies consider the personality traits in dermatologic diseases; however, few studies have focused on CSU and personality traits, especially in the Iranian population. Accordingly, the present study aimed to investigate the personality traits of patients with CSU compared to healthy cases.

\section{Methods}

In this cross-sectional study conducted during 2016 2018, all patients with CSU referred to asthma, and allergic diseases clinics of Ghaem Hospital were considered the research community. One hundred participants were selected using the convenience sampling method, and 100 healthy participants were selected as a control group. The inclusion criteria were the confirmed diagnosis of CSU based on the pathology findings and dermatologists' diagnosis and willingness to participation. Furthermore, patients with missing data, infectious diseases, other allergic diseases, auto-immune diseases, cancer, inflammatory diseases, and psychiatric disorders were excluded from the study.

The present study was approved by The Ethics Committee of the Mashhad University of Medical Sciences (code: IR.MUMS.fm.REC.1394.641). All patients and individuals in the control group participated voluntarily, and they were ensured of information confidentiality.

\subsection{Research Instrument}

The Temperament and Character Inventory (TCI) and a demographic checklist were used in this study. Cloninger developed the former to measure personality traits in seven scales, including novelty seeking, harm avoidance, reward dependence, persistence, cooperativeness, selfdirectiveness, and self-transcendence. The first four scales are associated with temperament, and the last three scales address character. This inventory is applied for individuals aged 18 years or above, and it has 240 questions with true or false responses (24). This inventory has been widely used in different communities and is also normalized in the Iranian population (25). Furthermore, the demographic checklist included demographic characteristics such as age, gender, marital status, level of education, and disease duration. Data were analyzed with SPSS Software version 23 using descriptive statistics, $t$-test, and chi-square test.

\section{Results}

The research sample size was calculated based on Alan et al.'s (26) study. This research encompassed 100 patients with CSU (34 males and 66 females) and 100 healthy individuals ( 33 males and 67 females). Regarding demographic characteristics, the patient group were $32.84 \pm 8.67$ years, while the healthy group were $30.9 \pm 7.55$ years. There was no significant difference between the studied groups considering gender and age $(\mathrm{P}=0.881, \mathrm{P}=0.97)$. In terms of marital status, the patient group included 58 married participants and 42 single participants, while 53 participants were married and 47 participants were single in the control group $(P=0.477)$. There was a significant difference among demographic variables in terms of level of education $(\mathrm{P}=0.001)$. In the patient group, 49 participants had a diploma or lower academic degree, and 51 participants had a master's or higher degree, while in the healthy group, 27 participants had a diploma or lower degree, and 73 participants had a master's or higher degree. The findings of the Cloninger inventory are presented in Table 1.

\begin{tabular}{lccc}
\hline \multicolumn{4}{l}{ Table 1. TCI Scores of Patient and Healthy Groups ${ }^{\mathrm{a}}$} \\
\hline Subscales & Patient Group & Healthy Group & P-Value $^{\text {b }}$ \\
\hline Novelty seeking & $9.43 \pm 2.88$ & $8.68 \pm 2.22$ & 0.04 \\
\hline Harm avoidance & $10.67 \pm 3.59$ & $9.49 \pm 3.22$ & 0.01 \\
\hline Reward dependence & $8.30 \pm 2.50$ & $8.39 \pm 2.30$ & 0.79 \\
\hline Persistence & $3.03 \pm 1.44$ & $2.90 \pm 1.43$ & 0.52 \\
\hline Self-directedness & $10.93 \pm 4.25$ & $12.81 \pm 4.72$ & 0.003 \\
$\begin{array}{l}\text { Self-transcendence } \\
\text { Cooperativeness }\end{array}$ & $9.08 \pm 2.99$ & $8.73 \pm 2.78$ & 0.39 \\
\hline $\begin{array}{l}\text { Abbreviation: TCI, Temperament and Character Inventory } \\
\text { a Values are expressed as mean } \pm \text { SD. } \\
\text { b Independent } t \text {-test was used. }\end{array}$ & 0.001 \\
\hline
\end{tabular}

The above table shows significant differences between the two groups in terms of novelty-seeking, harm avoidance, self-directedness, and cooperativeness. The patients with CSU had higher scores in novelty-seeking and harm avoidance, while the healthy individuals had higher scores in self-directedness and cooperativeness. Tables 2 and 3 show the TCI scores of the patient group by gender and marital status. As presented in Table 2, male and female patients differed in novelty-seeking, reward dependence, self-transcendence, and cooperativeness as male pa- 
tients had higher scores in novelty seeking and reward dependence, and female patients had higher scores in selftranscendence and cooperativeness. According to Table 3, the married patients had higher scores in harm avoidance, and the single patients had higher scores in persistence. In terms of age, there was no correlation between age and the TCI subscales in the patient group. While, there were correlations between disease duration with reward dependence, self-directedness, and self-transcendence.

\begin{tabular}{|c|c|c|c|}
\hline Subscales & Male & Female & P-Value $^{b}$ \\
\hline Novelty seeking & $10.52 \pm 3.14$ & $8.86 \pm 2.58$ & 0.006 \\
\hline Harm avoidance & $10.47 \pm 3.47$ & $10.77 \pm 3.67$ & 0.69 \\
\hline Reward dependence & $7.44 \pm 2.51$ & $8.74 \pm 2.40$ & 0.01 \\
\hline Persistence & $3.02 \pm 1.44$ & $3.03 \pm 1.45$ & 0.99 \\
\hline Self-directedness & $11.55 \pm 4.42$ & $10.60 \pm 4.16$ & 0.29 \\
\hline Self-transcendence & $7.67 \pm 2.88$ & $9.80 \pm 2.80$ & 0.001 \\
\hline Cooperativeness & $12.61 \pm 3.66$ & $14.54 \pm 3.90$ & 0.02 \\
\hline
\end{tabular}

Abbreviation: TCI, Temperament and Character Inventory

${ }^{\text {a }}$ Values are expressed as mean $\pm \mathrm{SD}$.

${ }^{\mathrm{b}}$ Independent $t$-test was used.

\begin{tabular}{lccc}
\hline \multicolumn{4}{l}{ Table 3. TCI Scores of Patient Group by Marital Status ${ }^{\mathrm{a}}$} \\
\hline Subscales & Single & Married & P-Value $^{\mathbf{b}}$ \\
\hline Novelty seeking & $10.07 \pm 3.05$ & $8.96 \pm 2.68$ & 0.06 \\
\hline Harm avoidance & $8.16 \pm 2.94$ & $10.77 \pm 3.67$ & 0.02 \\
\hline Reward dependence & $8.16 \pm 2.94$ & $8.39 \pm 2.66$ & 0.64 \\
\hline Persistence & $2.64 \pm 1.55$ & $3.31 \pm 1.30$ & 0.02 \\
\hline Self-directedness & $11.95 \pm 4.77$ & $10.18 \pm 3.70$ & 0.05 \\
\hline Self-transcendence & $9.33 \pm 2.61$ & $8.89 \pm 3.25$ & 0.47 \\
\hline Cooperativeness & $13.92 \pm 4.04$ & $13.86 \pm 3.85$ & 0.93 \\
\hline
\end{tabular}

Abbreviation: TCI, Temperament and Character Inventory

${ }^{\text {a }}$ Values are expressed as mean $\pm \mathrm{SD}$.

${ }^{\mathrm{b}}$ Independent $t$-test was used.

\section{Discussion}

In this research, 100 patients with CSU and 100 healthy participants were evaluated using TCI. The findings indicated that the patients with CSU had higher scores in novelty-seeking and harm avoidance, while the healthy individuals had higher scores in self-directedness and cooperativeness. Regarding the demographic variables, male and female patients differed in novelty-seeking, reward dependence, self-transcendence, and cooperativeness as male patients had higher scores in novelty-seeking and reward dependence. In comparison, female patients had higher scores in self-transcendence and cooperativeness. Moreover, there was no correlation between age and the TCI subscales in the patient group. In contrast, there were correlations between disease duration with reward dependence, self-directedness, and self-transcendence.

The inheritable temperament dimensions (namely harm avoidance, novelty seeking, reward dependence, and persistence) determine individual's responses to stress and coping with various kinds of emotions such as fear (harm avoidance), anger (novelty seeking), attachment (reward dependence), and ambition (persistence).

Considering the relationship between personality factors and CSU (27), the authors purpose CSU as a psychodermatological problem. However, few studies have used personality inventories in patients with CSU, especially in the Iranian population.

In a study conducted in Turkey, Alan et al. (26) evaluated 70 patients ( 52 females and 18 males) with chronic urticaria and 60 healthy subjects ( 37 females and 23 males) using the temperament-character inventory. The mean ages of the patients and the healthy subjects were 41.56 \pm 11.1 and $37.80 \pm 11.91$ years, respectively. The mean ages in these two groups were higher than the one in the present research. According to the findings, patients had higher scores in novelty-seeking and lower scores in selfdirectedness, cooperativeness, and reward dependence. This finding is the same as that of the present study. Although in our research, patients had a higher score in harm avoidance.

In another study by Pasaoglu et al., Minnesota Multiphasic Personality Inventory (MMPI) was used to evaluate personality traits in 59 patients with chronic spontaneous urticaria and 59 healthy cases. The findings revealed that the patients had higher scores in depression, hysteria, psychasthenia, schizophrenia, social introversion, paranoia, hypochondriasis, and psychopathic deviance compared to the healthy subjects. There was no correlation between the MMPI scores and disease duration. In our study, however, there were correlations between some TCI scales and disease duration. Although the research instrument was different in the two studies, the findings indicated a significant difference between the two patient and healthy groups as patients with chronic spontaneous urticaria had higher scores in psychopathologic domains than compared to the healthy group (28). Similarly, Staubach et al. assessed mental disorders in 111 patients with CSU. They noticed that $48 \%$ of the patients had at least one psychosomatic disorder and the most common anxiety disorders (29).

Some researchers evaluated patients with other chronic skin diseases such as psoriasis. In line with the 
findings of the present study, Ak et al. examined the personality of psoriasis patients and healthy cases and concluded that patients had higher scores in noveltyseeking, harm avoidance, reward dependence, and selftranscendence (30). According to the present study and the literature, personality traits may be strongly associated with chronic dermatological diseases such as CSU. Furthermore, psychological consultations may be helpful for such patients in clinical settings.

The limitations of the present study are small sample size and the study population selected from one region of the country. Further studies with a larger sample size across the country and the evaluation of severity index and its relationship with personality traits may provide better and more accurate results. Furthermore, further studies are also recommended to assess other psychiatric comorbidities.

\subsection{Conclusions}

According to the findings, the patients with CSU had higher scores in novelty-seeking and harm avoidance; however, the healthy individuals had higher scores in selfdirectedness and cooperativeness. Furthermore, a correlation was noticed between disease duration and the subscales of reward dependence, self-directedness, and selftranscendence.

\section{Acknowledgments}

The authors would like to acknowledge the Mashhad University of Medical Sciences for supporting this study.

\section{Footnotes}

Authors' Contribution: FFH and MKh contributed to the conception and design of the work. MKh and ZJ collected the patients. ME contributed to the analysis, and interpretation of the result. FFH and MK critically wrote and revised the manuscript. All authors gave final approval and agree to be accountable for all aspects of work ensuring integrity and accuracy.

Conflict of Interests: Authors declare no conflict of interests.

\section{Ethical Approval: IR.MUMS.fm.REC.1394.641.}

Funding/Support: Mashhad University of Medical Sciences funded this study.

Informed Consent: The researchers explained the research objectives, type of research intervention, voluntary participation, procedures and protocol, duration, risks and benefits, confidentiality, and right to refuse or withdraw before participation.

\section{References}

1. Brzewski PL, Spalkowska M, Podbielska M, Chmielewska J, Wolek M, Malec K, et al. The role of focal infections in the pathogenesis of psoriasis and chronic urticaria. Postepy Dermatol Alergol. 2013;30(2):77-84. doi: 10.5114/pdia.2013.34155. [PubMed: 24278052]. [PubMed Central: PMC3834683].

2. Kaplan AP. Clinical practice: Chronic urticaria and angioedema. $N$ Engl J Med. 2002;346(3):175-9. doi: 10.1056/NEJMcp011186. [PubMed: 11796852].

3. Hon KL, Leung AKC, Ng WGG, Loo SK. Chronic Urticaria: An Overview of Treatment and Recent Patents. Recent Pat Inflamm Allergy Drug Discov. 2019;13(1):27-37. doi: 10.2174/1872213X13666190328164931. [PubMed: 30924425]. [PubMed Central: PMC6751347].

4. Doong JC, Chichester K, Oliver ET, Schwartz LB, Saini SS. Chronic Idiopathic Urticaria: Systemic Complaints and Their Relationship with Disease and Immune Measures. J Allergy Clin Immunol Pract 2017;5(5):1314-8. doi: 10.1016/j.jaip.2016.11.037. [PubMed: 28302451]. [PubMed Central: PMC6377705].

5. Kay AB, Clark P, Maurer M, Ying S. Elevations in T-helper-2-initiating cytokines (interleukin-33, interleukin-25 and thymic stromal lymphopoietin) in lesional skin from chronic spontaneous ('idiopathic') urticaria. Br J Dermatol. 2015;172(5):1294-302. doi: 10.1111/bjd.13621. [PubMed: 25523947].

6. Kocaturk E, Zuberbier T. New biologics in the treatment of urticaria. Curr Opin Allergy Clin Immunol. 2018;18(5):425-31. doi: 10.1097/ACI.0000000000000466. [PubMed: 30015639].

7. Min TK, Saini SS. Emerging Therapies in Chronic Spontaneous Urticaria. Allergy Asthma Immunol Res. 2019;11(4):470-81. doi: 10.4168/aair.2019.11.4.470. [PubMed: 31172716]. [PubMed Central: PMC6557779].

8. Fadaee J, Khoshkhui M, Emadzadeh M, Hashemy SI, Farid Hosseini R, Jabbari Azad F, et al. Evaluation of Serum Substance P Level in Chronic Urticaria and Correlation with Disease Severity. Iran J Allergy Asthma Immunol. 2020;19(1):18-26. doi: 10.18502/ijaai.v19i1.2414. [PubMed: 32245317].

9. Lee N, Lee JD, Lee HY, Kang DR, Ye YM. Epidemiology of Chronic Urticaria in Korea Using the Korean Health Insurance Database, 2010-2014. Allergy Asthma Immunol Res. 2017;9(5):438-45. doi: 10.4168/aair.2017.9.5.438. [PubMed: 28677358]. [PubMed Central: PMC5500699].

10. Zuberbier T, Balke M, Worm M, Edenharter G, Maurer M. Epidemiology of urticaria: a representative cross-sectional population survey. Clin Exp Dermatol. 2010;35(8):869-73. doi: 10.1111/j.13652230.2010.03840.x. [PubMed: 20456386].

11. Kang MJ, Kim HS, Kim HO, Park YM. The impact of chronic idiopathic urticaria on quality of life in korean patients. Ann Dermatol. 2009;21(3):226-9. doi: 10.5021/ad.2009.21.3.226. [PubMed: 20523794]. [PubMed Central: PMC2861226].

12. Bewley A. The neglected psychological aspects of skin disease. BMJ. 2017;358:j3208. doi: 10.1136/bmj.j3208. [PubMed: 28684435].

13. Shah R, Bewley A. The importance of integrated psychological interventions and dedicated psychologists in dermatology. Clin Exp Dermatol.2014;39(3):428-30. doi:10.1111/ced.12282. [PubMed: 24635097].

14. Korabel H, Dudek D, Jaworek A, Wojas-Pelc A. [Psychodermatology: psychological and psychiatrical aspects of dermatology]. Przegl Lek. 2008;65(5):244-8. Polish. [PubMed: 18853651].

15. Zabolinejad N, Molkara S, Bakhshodeh B, Ghaffari-Nazari H, Khoshkhui M. The expression of serotonin transporter protein in the skin of patients with chronic spontaneous urticaria and its relation with depression and anxiety. Arch Dermatol Res. 2019;311(10):825-31. doi: 10.1007/s00403-019-01969-y. [PubMed: 31520274]. 
16. Kieć-Swierczyńska M, Dudek B, Krecisz B, Swierczyńska-Machura D, Dudek W, Garnczarek A, et al. [The role of psychological factors and psychiatric disorders in skin diseases]. Medycyna Pracy. 2006;57(6):551-5. Polish. [PubMed: 17533993].

17. Sahin BASSAK, Soydas E, Oguz S, IȘIK SELDA, ERTEKIN HÜLYA. Increased Pathological worry levels in patients with alopecia areata. J Clin Anal Med. 2017;8(1):10-3. doi: 10.4328/JCAM.4527.

18. Picardi A, Amerio P, Baliva G, Barbieri C, Teofoli P, Bolli S, et al. Recognition of depressive and anxiety disorders in dermatological outpatients. Acta Derm Venereol. 2004;84(3):213-7. doi: 10.1080/00015550410025264. [PubMed:15202838].

19. Kim TS, Pae CU, Jeong JT, Kim SD, Chung KI, Lee C. Temperament and character dimensions in patients with atopic dermatitis. J Dermatol. 2006;33(1):10-5. doi: 10.1111/j.1346-8138.2006.00002.x. [PubMed: 16469078].

20. Kilic A, Gulec MY, Gul U, Gulec H. Temperament and character profile of patients with psoriasis. J Eur Acad Dermatol Venereol. 2008;22(5):537-42. doi: 10.1111/j.1468-3083.2007.02460.x. [PubMed: 18331306].

21. Karaca S, Emul M, Kulac M, Yuksel S, Ozbulut O, Guler O, et al. Temperament and character profile in patients with essential hyperhidrosis. Dermatology. 2007;214(3):240-5. doi: 10.1159/000099589. [PubMed: 17377386].

22. Bahmer JA, Kuhl J, Bahmer FA. How do personality systems interact in patients with psoriasis, atopic dermatitis and urticaria? Acta Derm Venereol. 2007;87(4):317-24. doi: 10.2340/00015555-0246. [PubMed: 17598034].
23. Cloninger CR, Svrakic DM, Przybeck TR. A psychobiological model of temperament and character. Arch Gen Psychiatry. 1993;50(12):975-90. doi: 10.1001/archpsyc.1993.01820240059008. [PubMed: 8250684].

24. Cloninger C. Temperament and personality. Curr Opin Neurobiol. 1994;4(2):266-73. doi: 10.1016/0959-4388(94)90083-3.

25. Kaviyani H, Pour Naseh M. Validation and standardization of Cloninger temperament and character inventory in Iranian population. Tehran Univ Med J. 2005;2(63):89-98. Persian.

26. Alan S, Canan F, Karakas AA, Gecici N. Temperament and character profiles of patients with chronic idiopathic urticaria. Postepy Dermatol Alergol. 2015;32(3):167-72. doi: 10.5114/pdia.2015.48063. [PubMed: 26161056]. [PubMed Central: PMC4495117].

27. Chung MC, Kaminski ER. Posttraumatic Stress Disorder and Chronic Idiopathic URTICARIA: the Role of Coping and Personality. Psychiatr Q. 2019;90(1):47-62. doi: 10.1007/s11126-018-9599-y. [PubMed: 30264369].

28. Pasaoglu G, Bavbek S, Tugcu H, Abadoglu O, Misirligil Z. Psychological status of patients with chronic urticaria. J Dermatol. 2006;33(11):76571. doi: 10.1111/j.1346-8138.2006.00178.x. [PubMed: 17073991].

29. Staubach P, Dechene M, Metz M, Magerl M, Siebenhaar F, Weller K, et al. High prevalence of mental disorders and emotional distress in patients with chronic spontaneous urticaria. Acta Derm Venereol. 2011;91(5):557-61. doi: 10.2340/00015555-1109. [PubMed: 21597672].

30. Ak M, Haciomeroglu B, Turan Y, Lapsekili N, Doruk A, Bozkurt A, et al. Temperament and character properties of male psoriasis patients. J Health Psychol. 2012;17(5):774-81. doi: 10.1177/1359105311423863. [PubMed: 22104665]. 